\title{
ERGONOMIA NA CONCEPÇÃO DE INTERFACES GRÁFICAS DE SALAS DE CONTROLE AVANÇADAS DE REATORES NUCLEARES
}

\section{ERGONOMICS IN THE CONCEPTION OF GRAPHICAL INTERFACES OF NUCLEAR REACTORS ADVANCED CONTROL ROOMS}

\author{
M.Sc Douglas Vidal Teixeira ${ }^{1}$; Dr. Isaac José Antonio Luquetti dos Santos ${ }^{2}$; Dr. Fernando Toledo \\ Ferraz $^{3}$; Dr. Paulo Victor Rodrigues de Carvalho ${ }^{4}$ \\ ${ }^{1}$ Federal Fluminense University - UFF - Niterói - Brasil douglasvidal@producao.uff.br \\ ${ }^{2}$ Institute of Nuclear Engineering - IEN - Rio de Janeiro - Brasil luquetti@ien.gov.br \\ ${ }^{3}$ Federal Fluminense University - UFF - Niterói - Brasil fernando@latec.uff.br \\ ${ }^{4}$ Institute of Nuclear Engineering - IEN - Rio de Janeiro - Brasil paulov@ien.gov.br
}

\begin{abstract}
Resumo
A ergonomia de concepção pode ser definida como a elaboração de novos produtos, processos ou sistemas informatizados, enfatizando a aplicação dos conceitos ergonômicos, seguindo os padrões ergonômicos contidos nas normas, objetivando o desenvolvimento de sistemas seguros, funcionais, adaptáveis as necessidades dos usuários, propiciando o aumento da confiabilidade operacional e da segurança na realização das tarefas. O objetivo desse trabalho é propor e aplicar uma estrutura metodológica para avaliação e desenvolvimento de interfaces gráficas de salas de controle de reatores nucleares com enfoque centrado nos operadores, na atividade dos operadores e na tecnologia de ferramentas computacionais disponíveis para confecção dessas interfaces.
\end{abstract}

Palavras-chave: ergonomia, interface, salas de controle, reator nuclear.

\section{Introdução}

Uma sala de controle contém os sistemas e as instruções necessárias para controle das condições operacionais de uma planta industrial, de modo a assegurar o seu funcionamento e desligamento confiável e seguro, em situações normais e de acidentes (CNEN-NE 1.01). As salas de controle avançadas de plantas industriais são constituídas por um arranjo de sistemas, equipamentos, onde os operadores monitoram, controlam e intervêm no processo através de várias interfaces gráficas e estações de monitoramento. Essas interfaces apresentam implicações significativas para a segurança da planta, pois influenciam na atividade dos operadores, afetam o modo como os operadores recebem informações relacionadas com o status dos principais sistemas e determinam os requisitos necessários para que os operadores entendam e supervisionem os 
principais parâmetros. A tarefa principal do grupo de operação é manter a planta operando em condições aceitáveis de segurança e eficiência. Uma interface pode ser definida como parte de um sistema com o qual o usuário realiza contato através do plano físico, perceptivo e cognitivo. A interface é formada por apresentações de informações, de dados, de controles e de comandos em telas de computadores. Uma interface define as estratégias para a realização da tarefa, conduz, orienta, recepciona, alerta, ajuda e responde ao usuário durante as interações.

Segundo REASON (1990), os eventos de falhas humanas são influenciados pelos fatores internos e externos que modelam o desempenho humano (treinamento, estresse, condições ambientais, etc), fatores organizacionais e o projeto das interfaces homem sistema. Segundo HYMAN (1994), sistemas complexos com falhas no projeto das interfaces e na interação homem sistema podem contribuir para as falhas humanas. Os projetistas ainda não consideram no projeto de um sistema as características da tarefa, as necessidades do usuário, conforto e segurança, resultando em produtos ou postos de trabalho não adequados às características dos usuários e às tarefas por eles executadas. Embora alguns fabricantes de equipamentos já tenham percebido a necessidade de alocar os requisitos ergonômicos em seus produtos, é necessário que o projeto tenha uma abordagem centrada no usuário e no trabalho possibilitando a diminuição dos incidentes, acidentes, otimizando a eficiência da interação homem sistema e aumentando a confiabilidade operacional (SANTOS et al., 2004).

O objetivo desse trabalho é propor e aplicar uma estrutura metodológica para avaliação e desenvolvimento de interfaces gráficas de salas de controle de reatores nucleares com enfoque centrado nos operadores, na atividade dos operadores e na tecnologia de ferramentas computacionais disponível para confecção dessas interfaces. Esta estrutura metodológica é formada por três fases. A primeira fase consiste de um processo de avaliação integrada das interfaces gráficas existentes na sala de controle avançada do simulador compacto de um reator nuclear do tipo PWR (Pressurized water reactor), do laboratório de interfaces homem sistema, LABIHS, do Instituto de Engenharia Nuclear no Rio Janeiro. Na segunda etapa, os dados obtidos através desse processo de avaliação são utilizados no desenvolvimento das novas telas gráficas dos diversos sistemas que compõem o simulador. $\mathrm{Na}$ terceira fase estas novas interfaces serão avaliadas e os resultados comparados com a avaliação integrada realizada na primeira fase com as interfaces existentes. Este trabalho apresenta a estrutura metodológica utilizada e os resultados obtidos na primeira e segunda fase.

\section{Sala de Controle Avançada do Simulador de um Reator Nuclear PWR}

O simulador do Laboratório de Interfaces Homem-Sistema (LABIHS) localizado na Divisão 
de Instrumentação e Confiabilidade Humana (DICH) do Instituto de Engenharia Nuclear, IEN/CNEN, foi desenvolvido em conjunto com o Korean Atomic Energy Research Institute (KAERI) e consiste de uma rede de 9 computadores PC Pentium IV, que funcionam como terminais de uma estação de trabalho HP 3700, na qual opera o simulador compacto. O simulador é constituído essencialmente de cinco partes. A primeira é o programa de modelagem matemática da usina nuclear, programado em fortran, que contém em seu código a lógica do funcionamento da usina. A segunda parte é a memória compartilhada programada em $\mathrm{C} / \mathrm{C}++$, que tem como principal função armazenar as variáveis processadas pelo programa de modelagem matemática e disponibilizá-las para leitura e escrita, por outros programas. A terceira parte é a interface gráfica programada em $\mathrm{C} / \mathrm{C}++$ com bibliotecas ILOG, utilizando o software HSI Builder para a criação de suas telas e do programa ILOG Views Studio para a criação dos objetos gráficos utilizados na criação das telas. A quarta parte é o programa de controle do instrutor programado em $\mathrm{C} / \mathrm{C}++$, que controla a execução/interrupção do programa de modelagem matemática, salva/carrega/altera o estado da usina simulada e é capaz de inserir erros com tempo programado na simulação para testar a reação dos operadores. A quinta parte é a base de dados, que contém e disponibiliza dados estáticos para os programas, como por exemplo os necessários na inicialização do programa de modelagem matemática. Todas as cinco partes que compõem o simulador são executadas na estação de trabalho HP, que roda o sistema operacional HP-UX multiusuário.

O grupo de operação da sala de controle do simulador é constituído por três operadores: operador do primário, operador do secundário e um supervisor. Cada operador controla e monitora os sistemas sob sua responsabilidade, através de três telas coloridas de computador do tipo LCD de 18 polegadas, associadas com um teclado e um mouse. Em uma das paredes da sala de controle também está instalado um telão, que apresenta o funcionamento geral da planta nuclear e dos sistemas. Este telão tem como finalidade propiciar ao operador uma visão integrada do funcionamento do reator. Em uma sala anexa à sala de operação atua o instrutor, que programa os eventos que serão simulados. A operação do simulador é feita através da navegação nessas telas coloridas, que representam os principais sistemas do reator nuclear PWR de três loops. O controle e monitoração do simulador são realizados através dos sistemas apresentados nas seguintes telas: tela do sistema de refrigeração do reator; tela do sistema de controle químico e volumétrico; tela do sistema de remoção do calor residual; tela do sistema de vapor principal e sistema da turbina; tela do sistema de água de alimentação; tela do sistema do condensador; tela do sistema elétrico; tela do sistema de controle das barras do reator; tela do sistema de controle da reatividade; tela com o arranjo das barras de controle e desligamento; tela com a descrição das mensagens de alarme; tela de anunciação alarme 1; tela de anunciação alarme 2; tela do status dos permissivos; tela com os gráficos representando a tendência das variáveis e tela de overview da planta nuclear. 
A identificação do nome do sistema está localizada na parte superior de cada tela. A área gráfica está posicionada na parte central de cada tela. Existem quatro modos de navegação entre as telas. No lado direito da área gráfica central existem dez teclas, que permitem a navegação entre os sistemas. No lado direito da área gráfica central existem quatro setas, que permitem navegar entre as telas dos sistemas. Além dessas quatro setas, existem também duas teclas que apresentam o histórico das telas acessadas e a última tela apresentada, ou seja, teclas HISTORY e PREVIOUS. Para navegar entre as telas pode-se também clicar no link que conecta uma tela de um sistema com a tela de outro sistema. Outro modo de navegação é feito através da digitação do nome da tela requerida. Esta digitação é realizada no espaço reservado na tela para este comando. Este espaço está localizado no lado direito inferior da tela. A figura 1 apresenta estas informações.

Figura 1: Tela do sistema de refrigeração do reator

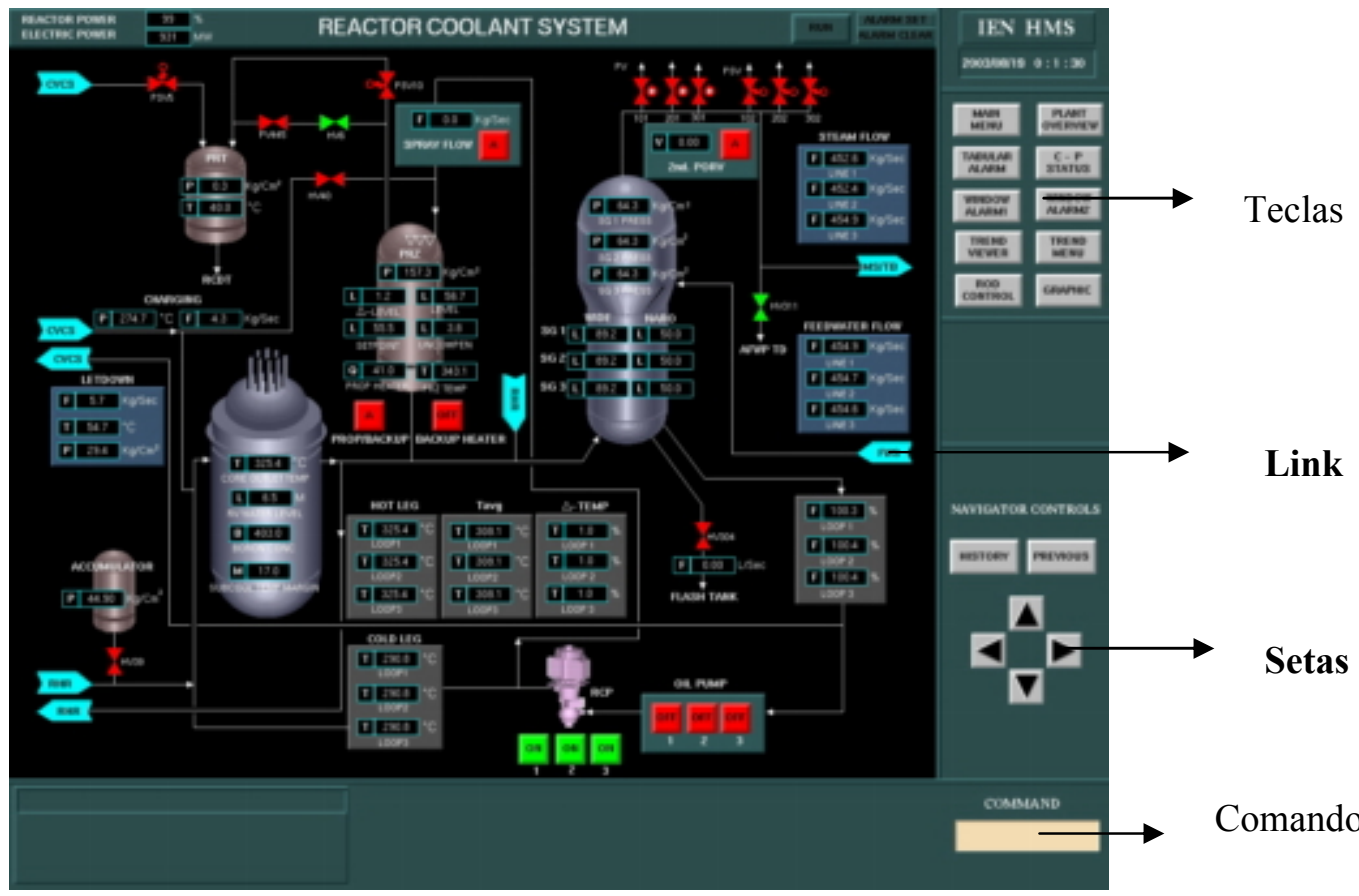

\section{Estrutura Metodológica}

A estrutura metodológica é apresentada na figura 2. Neste trabalho apresentamos as etapas relacionadas com a análise das tarefas, avaliação integrada (normas e guias de fatores humanos, lista de verificação, entrevista, análise da atividade dos operadores) e desenvolvimento das interfaces. 
Figura 2: Estrutura metodológica

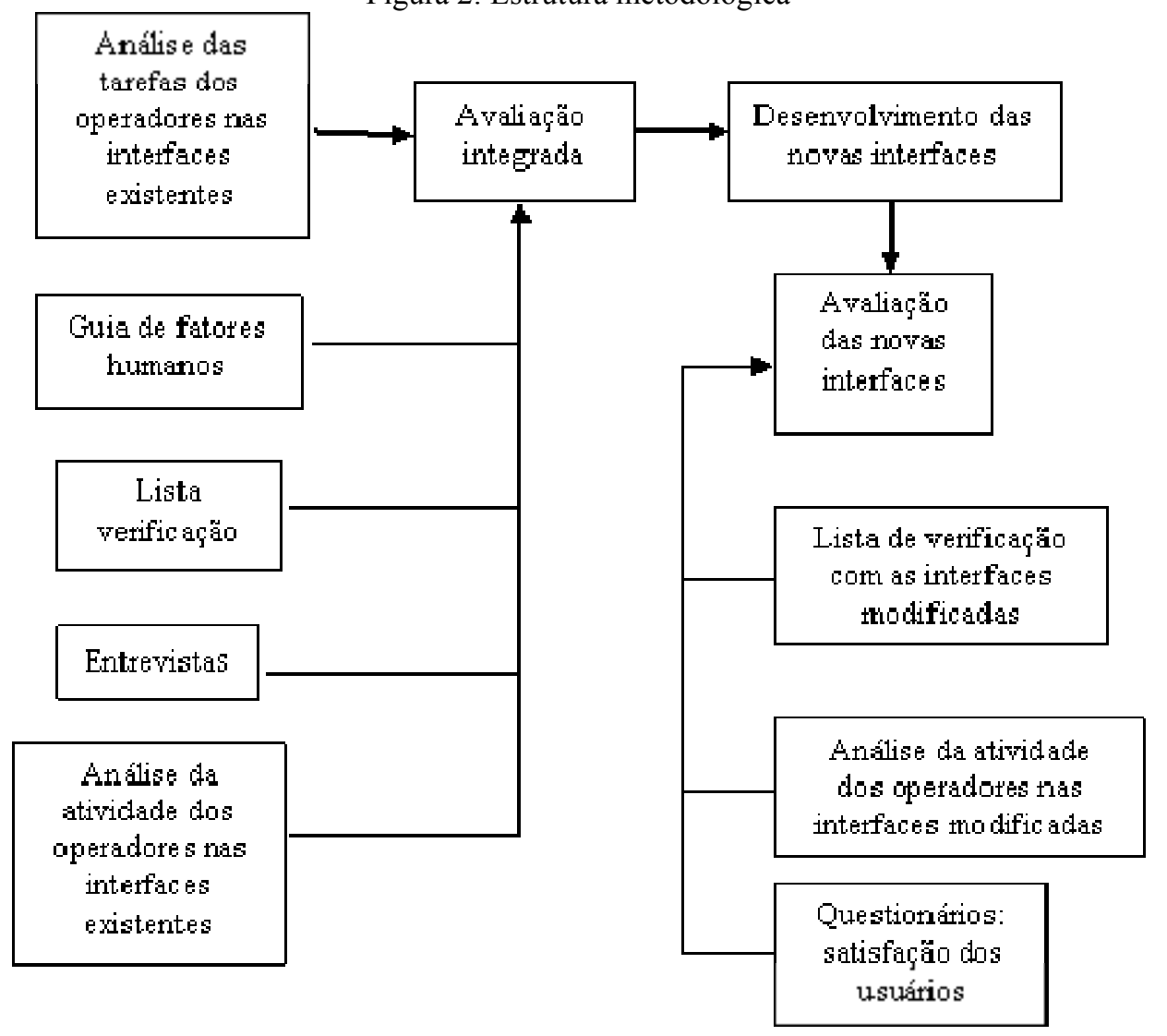

\subsection{Análise das tarefas}

A análise das tarefas é focada no aspecto prescrito das tarefas, nas ações que devem ser realizadas pelo operador, nos objetivos que ele tem de alcançar, nos recursos e condições de que dispõe. Segundo MEISTER (1985), a análise das tarefas prioriza as informações requeridas pelos operadores, as informações disponíveis, como os operadores avaliam essas informações, as ações realizadas pelos operadores, a freqüência com que cada ação é realizada, as ferramentas necessárias. Neste trabalho foi implementada a análise das tarefas dos operadores com relação aos procedimentos de emergência, após o desligamento do reator, para identificação dos seguintes acidentes postulados: rompimento do tubo de um gerador de vapor (SGTR-Steam Generator Tube Rupture) e perda do refrigerante (LOCA-Loss Coolant Accident).

\subsection{Guias de fatores humanos}

A Comissão Reguladora da Área Nuclear nos Estados Unidos da América implementou em 1991 o guia NUREG 700, que incluiu no projeto de salas de controle e interfaces os requisitos relacionados com fatores humanos. Em 2002 a NUREG 700 foi atualizada e dividida em quatro seções. A seção um apresenta os seguintes elementos, que devem ser incluídos no projeto das interfaces operador sistema: apresentação das informações, interação operador sistema e controles. 
A seção dois apresenta os seguintes sistemas: sistema de alarme, sistema de auxílio ao operador, sistema computadorizado dos procedimentos de operação, sistema de comunicação, sistema computadorizado de controle, sistema de monitoração das funções de segurança. A seção três apresenta o projeto das estações de trabalho e do local de trabalho. A avaliação foi baseada nas recomendações da NUREG 700 e abrangeu somente os aspectos ergonômicos relacionados com o projeto das interfaces, ou seja, apresentação das informações, interação, controles, navegação.

\subsection{Listas de verificação}

Listas de verificação são ferramentas utilizadas para verificar se o projeto possui determinados atributos considerados desejáveis e importantes. As listas de verificação estabelecem um método revisor estruturado com o objetivo de assegurar que o projeto atingiu determinados critérios, estabelecendo uma comparação com os padrões desejados. Elas devem indicar de maneira clara e exata as informações que estão sendo procuradas. Foi desenvolvida e utilizada uma lista de verificação, a partir das listas apresentadas por SANTOS (2003). A lista de verificação consiste de um questionário de 72 questões objetivas com pontuação mínima de 1 ponto e máxima de 9. Cada característica é avaliada em uma escala de 3 posições que varia de 1 a 3, conforme mostrado abaixo:

3 = A característica avaliada está completamente de acordo.

2 = A característica avaliada está parcialmente de acordo.

1 = A característica avaliada não está de acordo.

Desde que algumas características possam ser mais importantes do que outras, os pesos de importância podem ser atribuídos. Cada característica é avaliada segundo sua importância em uma escala que vai de 1 a 3, conforme mostrado abaixo:

3 = A característica avaliada é imperativa.

2 = A característica avaliada é desejável.

1 = A característica avaliada não é importante

Pontuação final = Avaliação X Importância

\subsection{Entrevistas}

$\mathrm{Na}$ pesquisa qualitativa a entrevista caracteriza-se como um instrumento importante para a produção de informações fornecidas diretamente pelos sujeitos envolvidos no processo. Dessa forma, a entrevista como fonte de informações pode ser estruturada, semi-estruturada ou não 
estruturada. As entrevistas não estruturadas são projetadas para permitir ao especialista que discuta o problema a ser resolvido de modo natural. Assim é possível obter a compreensão dos conceitos mais importantes e conhecer as estratégias que o especialista utiliza para resolver o problema. A desvantagem das entrevistas não estruturadas é uma grande quantidade de informações fragmentadas. A entrevista estruturada mantém o foco do problema a ser resolvido e adquire detalhes específicos a respeito de determinado aspecto do problema antes de passar para outros pontos (EYDE, 1993). A desvantagem principal é a fraca compreensão das regras ou estratégias para solucionar problemas.

Em nosso processo utilizamos a forma semi-estruturada, individual e coletiva, combinando perguntas fechadas e abertas, com o objetivo de possibilitar ao operador a oportunidade de se pronunciar sobre o funcionamento do simulador e principalmente das interfaces. Estabelecemos um roteiro de questões, privilegiando aspectos que julgamos importantes de serem coletados, no momento da entrevista, deixando em aberto a possibilidade das questões previamente formuladas serem desdobradas, de acordo com as informações repassadas durante a mesma. O roteiro previa questões relacionadas com a facilidade de operação, questões de navegação, mensagens de alarmes, compreensão do processo, disponibilidade dos procedimentos, compreensão dos componentes e uniformidade nas unidades.

As entrevistas foram realizadas no local de trabalho, por considerarmos o ambiente de trabalho o local mais apropriado para que os operadores falem sobre suas atividades, suas experiências e seus conhecimentos. Participaram das discussões individuais e coletivas, não só os operadores, mas também o engenheiro responsável pelo LABIHS e o engenheiro responsável pela área de fatores humanos e ergonomia.

\subsection{Análise da atividade dos operadores}

Para analisar a atividade dos operadores da sala de controle avançada do simulador do laboratório de interfaces homem sistema, LABIHS, com as interfaces existentes, foram realizadas observações sistemáticas para dois tipos de acidentes postulados: rompimento do tubo de um gerador de vapor (SGTR-Steam Generator Tube Rupture) e perda do refrigerante (LOCA-LosS Coolant Accident). Acidentes postulados são acidentes considerados como de ocorrência admissível para fins de análise, visando ao estabelecimento das condições de segurança, capazes de impedir e/ou minimizar eventuais conseqüências (CNEN-NE-1.04).

Durante uma situação de acidente postulado, ocorrem múltiplos alarmes, levando ao desligamento automático do reator ou tornando necessário a atuação do operador para desligamento seguro da planta nuclear. Após o desligamento, é necessário que os operadores realizem ações padrões e utilizem procedimentos de emergência, com o objetivo de identificar qual tipo de acidente 
postulado ocorreu. Após essa identificação, os operadores aplicam procedimentos de recuperação de modo a manter o reator seguro, mesmo após seu desligamento. O registro das informações abrange desde a fase de ocorrência dos múltiplos alarmes até a identificação do tipo de acidente postulado. Esses registros foram obtidos através de gravações de áudio e vídeo, anotações escritas e a observação dos $\log s$ gerados pelo simulador. Os $\log s$ são os registros da operação realizada no sistema, quantificando a quantidade de interfaces visitadas, número de controles atuados e o tempo gasto na operação.

O objetivo nesta fase é obter um entendimento mais detalhado da interação operadores sistemas, ou seja: navegação, tarefas completadas corretamente, tempo para realizar cada tarefa, erros cometidos, se os usuários receberam realimentação adequada em cada operação, se os usuários identificaram problemas no menu do sistema e se o menu facilita o entendimento da seqüência correta de operação.

\subsection{Desenvolvimento das interfaces}

O desenvolvimento das novas interfaces teve o auxílio das seguintes ferramentas computacionais: o HSIBuilder (2002), o ILOG Views Studio (2000), o Borland C++ Builder (BORLAND, 2006) e o Humminbird Neighbourhood (Hummingbird Connectivity 2006). O HSIBuilder é um aplicativo de desenvolvimento de interfaces que permite que uma tela seja carregada com uma determinada visualização gráfica, podendo ser alterada quanto à sua apresentação visual (tamanho, cor, etc) e quanto às suas propriedades.

O ILOG Views Studio é a principal ferramenta para a construção e desenvolvimento de aplicativos, bibliotecas de protótipos gráficos, interfaces e scripts de utilização. Neste trabalho o ILOG Views Studio foi utilizado para a construção de novos componentes e alteração das propriedades de aplicativos, como por exemplo, o tamanho e formato das telas das interfaces.

O Borland $\mathrm{C} / \mathrm{C}++$ Developer's Studio foi utilizado juntamente com o Humminbird Neighbourhood para as alterações em código que tiveram de ser realizadas nas interfaces. Foi desenvolvido um novo sistema constituído de novas interfaces, que pode ser executado em paralelo com o sistema original, onde as telas que não foram alteradas continuam sendo carregadas juntamente com as telas, o layout e os objetos adicionais que foram criados. Não foram realizadas alterações no programa de modelagem matemática da usina nuclear, programado em fortran, que contém em seu código a lógica do funcionamento da usina. 


\section{Resultados}

Através do guia de fatores humanos, NUREG 700, das entrevistas semi-estruturadas, das listas de verificações e da análise da atividade dos operadores, utilizando as interfaces existentes, um conjunto de informações e dados foi obtido propiciando o desenvolvimento das novas interfaces. A seguir citamos algumas destas informações e dados:

- os componentes controlados pelos operadores, ou seja, bombas, acionadores, devem ser identificados de modo diferente dos componentes que não são controlados, facilitando a operação. Por exemplo, através de cores diferentes;

- ocorrência de problemas com a apresentação das legendas. Algumas legendas estavam em negrito e outras fora de lugar, ou seja, sem uma posição padronizada nas telas. Outro problema foi a não uniformização das unidades de algumas variáveis;

- redução no número de telas percorridas, evitando a passagem pela tela MAIN MENU que dá acesso as outras telas do simulador;

- o sistema de alarme não facilita a tomada de decisão do operador quando uma resposta rápida é exigida. A avaliação indicou que as atuais mensagens dos alarmes não estavam esclarecendo o evento ocorrido quanto à sua localização, causa e efeito. Neste caso, a alteração feita foi na troca das strings de mensagens;

- alteração das telas de controle popup com a padronização do posicionamento, criação no novo layout de uma área maior para comportar as telas de controle, modificação na propriedade de cada item que possui controle para exibir sua respectiva tela extra na área de controle do layout, alteração da disposição dos itens das telas de controle para ocuparem uma nova área do layout, alteração no código para permitir animação das telas de controle dentro do espaço do layout;

- a tecla HISTORY não atualizava as telas visitadas pelo operador durante a navegação. Isto causa um problema no momento em que o botão PREVIOUS era clicado. Como o histórico não retratava as telas navegadas na ordem real, o botão PREVIOUS nem sempre realizava a ação esperada, não esclarecendo o operador a ordem de navegação. O objetivo desta alteração é fazer com que o histórico atualize todas as telas na ordem com que elas realmente são navegadas;

- o novo layout foi criado baseado nas deficiências apontadas pela avaliação. Foi verificado que os botões laterais de navegação não estavam sendo utilizados pelos operadores. Também foi visto que havia muito espaço nas telas não utilizado. Como as telas deveriam conter as opções de controle, que antes eram destinadas às janelas de popup, a área também foi aumentada. Foi possível colocarmos botões da navegação para todas as telas pertencentes ao sistema. Um grupo de telas foi colocado em uma área específica por pertencerem a um grupo funcional distinto e de pouco acesso. Os botões de histórico (HISTORY), o de retorno (PREVIOUS) e o campo para entrada de comando 
diretamente pelo teclado foram mantidos. A área lateral pôde ser diminuída, aumentando o tamanho útil das telas do simulador;

- a navegação entre as telas do sistema estava com diversas falhas de continuidade. Links que apontavam para determinadas telas não encontravam os seus respectivos pares quando clicados. Isto causava confusão e inconsistência no sistema, tornando a navegação muitas vezes confusa para os operadores inexperientes. O número de links presentes nas telas aumentou consideravelmente demonstrando uma falha que existia no sistema original. Entretanto, a complexidade visual das telas aumentou consideravelmente, porém os benefícios na otimização da navegação tornou a representação do processo para os operadores mais eficiente, otimizando as tomadas de decisão;

- criação da nova tela Main Menu: com a criação do novo layout a tela de abertura perderia o seu principal sentido na operação, que era de servir de link para todas as outras telas do sistema. Como isso agora é feito diretamente no layout, tornou-se necessária uma nova utilidade para a tela Main Menu.

A figura 3 apresenta o layout das novas interfaces. A figura 4 apresenta a nova tela Main Menu.

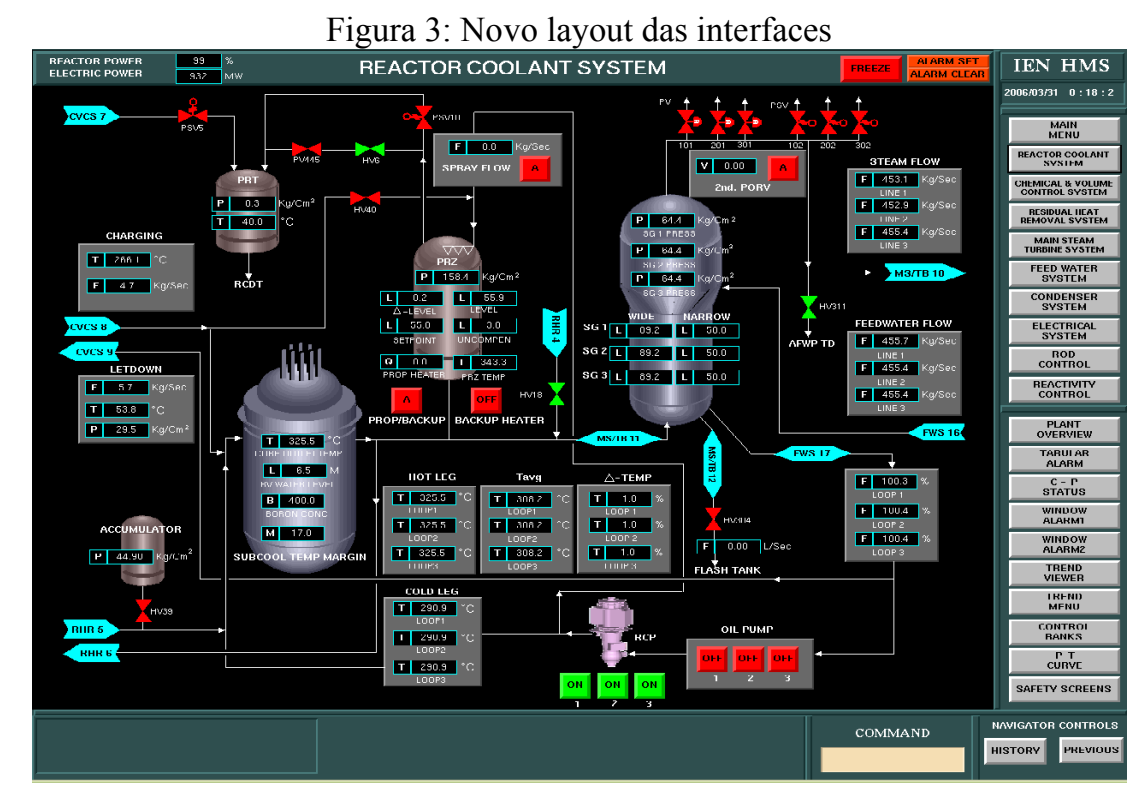


Figura 4: Nova tela Main Menu

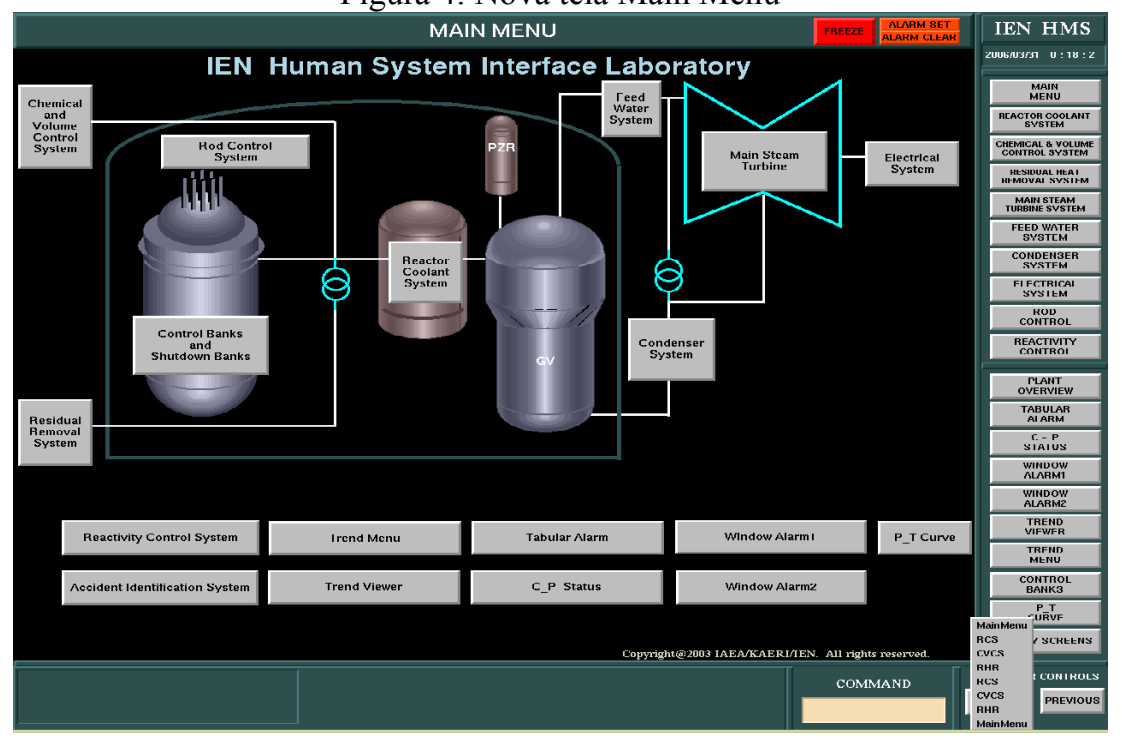

\section{Conclusões}

O desenvolvimento das novas interfaces foi centrado nos operadores, na atividade dos operadores e na tecnologia de ferramentas computacionais disponível para confecção dessas interfaces. A primeira fase consistiu de um processo de avaliação integrada das interfaces gráficas existentes utilizando as seguintes ferramentas: entrevistas semi-estruturadas, norma e guia de fatores humanos, NUREG 700, listas de verificação e a análise da atividade dos operadores. Na segunda etapa, os dados obtidos através desse processo de avaliação foram utilizados no desenvolvimento das novas telas gráficas dos diversos sistemas que compõem o simulador. $\mathrm{Na}$ terceira fase estas novas interfaces serão avaliadas e os resultados comparados com a avaliação integrada realizada na primeira fase. A estrutura metodológica utilizada possibilitou a avaliação do modo de navegação e interação dos operadores com as telas dos sistemas, o formato e as informações disponibilizadas para os operadores nas telas e o sistema de alarme.

Os dados apresentados mostram que a análise da atividade dos operadores no ambiente de emergência simulado complementa a avaliação através de listas de verificação, normas e guias de fatores humanos contribuindo com informações para a melhoria das telas do simulador, mostrando as dificuldades na identificação de quais componentes da planta podem ser controlados e na falta de uma hierarquização dos alarmes, seguindo um critério de prioridades para segurança da usina.

Foi observado que a atitude dos operadores quando se deparavam com a situação de não conseguir controlar determinado componente da planta, está relacionada com a falta de treinamento específico. Essa falta de treinamento também foi constatada pela pouca experiência com os procedimentos. Estes que não podem dar margem as dúvidas, se encontram em inglês, dificultando 
o aprendizado dos operadores. Finalmente, o sistema de alarme que durante os períodos de alta carga de trabalho deveria prover a necessária assistência aos operadores, não auxilia na tomada de decisões, tornando o diagnóstico mais difícil.

\begin{abstract}
The ergonomics of conception can be defined as the elaboration of new products, processes, work methods or computational systems, emphasizing the application of the ergonomics requirements, following the ergonomics principles contained in the norms, objectifying the development of safety systems, adaptable with the user necessities, propitiating the increase of the operational reliability and the safety in the accomplishment of the tasks. The objective of this paper is to apply a structure methodological to evaluate and develop new interfaces of nuclear control rooms with approach centered in the operators, the operators activity and the available technology of computational tools.
\end{abstract}

Key-words: ergonomics, interface, control room, nuclear reactor

\title{
Referências
}

CNEN-NE-1.01, Comissão Nacional de Energia Nuclear. Licenciamento de Operadores de Reatores Nucleares.

CNEN-NE-1.04, Comissão Nacional de Energia Nuclear. Licenciamento de Instalações Nucleares

Eyde, L. D., Robertson, G. J., Krug, S. E. Responsible Test Use: Case Studies For Assessing Human Behaviour. Washington DC: American Psychological Association, 1993.

cross ${ }^{\text {ref }}$

Hyman, W. A. Errors in The Use of Medical Equipment. Human Error in Medicine. Lawrence Erlbaum Associates, Inc. London, 1994.

Meister, D. Human Factors in Reliability, New York, Mc Graw Hill, 1990.

NUREG 700 (Revision 2), U.S. Nuclear Regulatory Commission (NRC). Human System Interface Design Review Guideline, 2002

Reason, J. Human Error. Cambridge University, 1990

Santos, I. J. A. L. e Vidal, M. C. A Ergonomia no Licenciamento e Avaliação de Salas de Controle de Reatores Nucleares. COPPE/UFRJ, 2003.

Santos, I. J. A. L., Silva, C. B., Carvalho, P. V. e Vidal, M. C. Usability as Evaluation Tool for Nuclear Medical Equipment. XXII Congresso Brasileiro de Medicina Nuclear, Aracaju, Sergipe, 2004.

\section{Inserir aqui dados completos de TODOS os autores:}

Nome completo: Douglas Vidal Teixeira

Filiação institucional: Pontifícia Universidade Católica do Rio de Janeiro; PUC-RIO (atual)

Departamento: CETUC - Centro de Estudos em Telecomunicações

Função ou cargo ocupado: Doutorando 
Endereço: Rua Joaquim Távora 148/1001, Icaraí, Niterói, RJ; CEP: 24230-540. Brasil.

Telefones para contato: 212710 2803; 2199725199

e-mail:1ouglasvidal@producao.uff.br

Nome completo: Fernando Toledo Ferraz

Filiação institucional: Universidade Federal Fluminense (UFF)

Departamento: TEP - Departamento de Engenharia de Produção

Função ou cargo ocupado: Professor

Endereço: Rua Cesario Alvim, 37/105/b.01, humaita, Rio de Janeiro, RJ. Brasil.

Telefones para contato: 21 2629-5711 - 96333995

e-mail:fernandoferrazuff@yahoo.com

Nome completo: Isaac José Antonio Luquetti dos Santos

Filiação institucional: Instituto de Engenharia Nuclear - IEN

Departamento: CNEN - Centro de Estudos em Energia Nuclear

Endereço: Comissão Nacional de Energia Nuclear, Instituto de Engenharia Nuclear, Coordenação de Instrumentação. Cidade Universitária - Ilha do Fundão - Ilha do Governador 21945-970 - Rio de Janeiro, RJ - Brasil - Caixa-Postal: 68550

Telefones para contato: (21) 22098196

e-mail:luquetti@ien.gov.br

Nome completo: Paulo Victor Rodrigues de Carvalho

Filiação institucional: Instituto de Engenharia Nuclear - IEN

Departamento: CNEN - Centro de Estudos em Energia Nuclear

Endereço: Comissão Nacional de Energia Nuclear, Instituto de Engenharia Nuclear, Divisão de

Instrumentação e Confiabilidade Humana.

Cidade Universitária - Ilha do Fundão

Ilha do Fundão

21945-970 - Rio de Janeiro, RJ - Brasil - Caixa-Postal: 68550

Telefones para contato: (21) 25604113 Ramal: 2214

e-mail: paulovien gov.br

Recebido para publicação em: 03/04/07

Aceito para publicação em: 09/05/07 